\title{
Trypanosoma cruzi in Nonischemic Cardiomyopathy Patients, Houston, Texas, USA
}

Melissa S. Nolan, David Aguilar, ${ }^{1}$ Arunima Misra, ${ }^{2}$ Sarah M. Gunter, Tim Erickson, Rodion Gorchakov, ${ }^{3}$ Hilda Rivera, Susan P. Montgomery, Kristy O. Murray

To investigate possible cardiac manifestations of Chagas disease, we tested 97 Latinx patients with nonischemic cardiomyopathy in Houston, Texas, USA, for Trypanosoma cruzi infection. We noted a high prevalence of underdiagnosed infection and discrepant results in clinical diagnostic assays. Latinx cardiac patients in the United States would benefit from laboratory screening for $T$. cruzi infection.

$\mathrm{T}$ he clinical manifestations of Chagas disease, caused by infection with the Trypanosoma cruzi parasite, are cardiac in approximately one third of patients. Without treatment, the parasite alternates between the trypomastigote and amastigote forms and causes direct smooth muscle tissue damage, myocardial fibrosis, chronic activation of inflammatory pathways, and autonomic dysfunction (1). This process can lead to progressive heart failure years later for some patients. Chagas cardiomyopathy patients can seek treatment for malignant ventricular arrhythmias, aneurysms, thromboembolism, or sudden cardiac death (2). Despite advances in our understanding of the pathogenic pathways, why some patients have onset of progressive cardiac disease whereas others remain in a persistent subclinical indeterminate disease remain unknown. Identifying infection status early, before the onset of heart failure, is critical because chemotherapeutics are most efficacious in the acute and early stages of infection.

\footnotetext{
Author affiliations: University of South Carolina, Columbia, South Carolina, USA (M.S. Nolan), Baylor College of Medicine, Houston, Texas, USA (M.S. Nolan, D. Aguilar, A. Misra, S.M. Gunter,

T. Erickson, R. Gorchakov, K.O. Murray), Harris Health System-Ben Taub Hospital, Houston (A. Misra), Centers for Disease Control and Prevention, Atlanta, Georgia, USA (H. Rivera,

S.P. Montgomery)
}

DOI: https://doi.org/10.3201/eid2707.203244
In the United States, $\approx 300,000$ persons are infected with $T$. cruzi parasites (3), and $<1 \%$ have received treatment (4). Because of low physician awareness (5), Chagas disease often is underdiagnosed or misdiagnosed. Previous cardiac patient seroprevalence studies in New York, NY, and Los Angeles, CA, suggest that the rate of undiagnosed $T$. cruzi infection is particularly high (13\%-19\%) among Latin American immigrants with dilated cardiomyopathy $(6,7)$. However, the extent of T. cruzi infection in the United States beyond these 2 metropolitan areas is largely unknown. We assessed the utility of T. cruzi diagnostic surveillance for Latinx patients with nonischemic cardiomyopathy who sought clinical care in a large tertiary care facility in Houston, Texas, USA.

\section{The Study}

During August 2015-July 2017, we recruited cardiac patients for Chagas disease surveillance from Harris Health System-Ben Taub Hospital, a large countyfunded tertiary care facility in Houston. Patients with known nonischemic cardiomyopathy who sought treatment at the outpatient cardiac clinic or who were admitted to a cardiac inpatient unit were invited to participate in our study. Inclusion criteria required a recorded ejection fraction $<50 \%$ within the past year and a recent negative ischemic work-up based on stress echocardiography or invasive coronary angiography. We excluded patients of non-Latinx ethnicity and those who were currently incarcerated, had prior T. cruzi serologic testing, had evidence of acute coronary syndrome suspected to be of Takotsubo

${ }^{1}$ Current affiliation: University of Texas Health Science Center, Houston, Texas, USA.

${ }^{2}$ Current affiliation: Michael E. Debakey Veterans Affairs Medical Center, Houston, Texas, USA.

${ }^{3}$ Current affiliation: King Abdullah University of Science and Technology, Thuwal, Saudia Arabia. 
origin, or had documentation of an alternative etiology for their nonischemic cardiomyopathy (e.g., peripartum, genetic, or alcoholic cardiomyopathy). Consent forms were available in English and Spanish, and licensed translators ensured that all potentially eligible participants were invited to participate. This protocol was reviewed and approved by the Baylor College of Medicine Institutional Review Board (protocol no. H-36761).

After consent, participating patients provided a blood sample for T. cruzi diagnostic testing and completed a risk factor questionnaire. The 5-page questionnaire was administered by a study team member and included sections on residential and travel histories, potential triatomine exposures and sources, current health symptoms and health behaviors, clinical family history, and knowledge, attitudes, and practices regarding Chagas disease. Initial T. cruzi diagnostic testing included T. cruzi-specific antibody testing using Chagas STAT-PAK Assay (Chembio Diagnostic Systems, Inc., https://chembio.com) and Hemagen Chagas Kit (Hemagen Diagnostics, Inc., https:/ / www.hemagen.com). Confirmation of positive and discordant results were then performed by using Chagatest ELISA Recombinante 3.0 (Wiener Laboratorios S.A.I.C., https://www.wiener-lab. com) and TESA blot by the Centers for Disease Control and Prevention.

During the 2-year study period, 97 patients with nonischemic cardiomyopathy were enrolled out of 132 eligible patients; 35 refused to participate because of lack of interest. The average age of participants was
52 years (range $28-91$ years); $38 \%$ of participants were female and $62 \%$ male. Birth countries for the cohort were Mexico (53\%), United States (14\%), El Salvador (12\%), Honduras (9\%), Guatemala (4\%), and other Latin America Spanish-speaking countries (8\%). Patients born in the United States originated from Texas $(n=9)$, New York $(n=2)$, Indiana $(n=1)$, and Oregon $(n=1)$. Of the cohort, $43 \%$ reported having previously seen the triatomine vector; 20/42 (48\%) reported sightings in Texas, compared with $31 / 42(74 \%)$ in a Chagas-endemic Latin American country. Furthermore, $12 \%$ of the cohort reported a history of triatomine bites. Despite high triatomine recognition, only $8 \%$ of the patient cohort had ever heard of Chagas disease, and only half of these patients could correctly state how Chagas disease is acquired.

Overall, 7\% of Latinx nonischemic cardiomyopathy patients seeking treatment for heart failure management were confirmed positive for $T$. cruzi infection by Centers for Disease Control and Prevention Wiener EIA and TESA blot confirmation testing. Discordant test results were common (Table), complicating the clinical decision-making process. All 7 patients who had laboratory-confirmed Chagas cardiomyopathy were born in a Latin America country: El Salvador $(n=4)$, Honduras $(n=1)$, Mexico $(n=1)$, and Venezuela $(n=1)$. All 7 confirmed positive patients had mothers who were born in or had lived in a Latin America country. Three had lived in a house with a dirt floor and 2 with a palm leaf thatched roof, which are both known risks for triatomine infestations $(8,9)$. One participant had received a blood transfusion in

Table. Characteristics of patients enrolled in a cross-sectional study of Trypanosoma cruzi infections in Latinx cardiomyopathy patients at a tertiary care facility† and results of 4 diagnostic assays, Houston, Texas, USA, 2015-2017*

\begin{tabular}{|c|c|c|c|c|c|c|c|}
\hline \multirow[b]{2}{*}{ ID } & \multirow[b]{2}{*}{ Age, y/sex } & \multirow[b]{2}{*}{ State, country of birth } & \multirow{2}{*}{$\begin{array}{c}\text { True } \\
\text { positive†§ }\end{array}$} & \multicolumn{2}{|c|}{ BCM testing } & \multicolumn{2}{|c|}{ CDC testing } \\
\hline & & & & Stat-Pak & Hemagen & Weiner EIA & TESA blot \\
\hline CM-013 & $79 / \mathrm{F}$ & Guerrero, Mexico & No & Faint positive & - & - & NP \\
\hline CM-014 & $66 / \mathrm{M}$ & La Union, El Salvador & Yes & + & + & + & + \\
\hline CM-017 & $62 / \mathrm{M}$ & San Salvador, El Salvador & Yes & + & + & + & + \\
\hline CM-037 & $73 / F$ & El Salvador $\ddagger$ & Yes & Faint positive & - & + & + \\
\hline CM-048 & $54 / \mathrm{M}$ & Texas, USA & No & Faint Positive & - & - & NP \\
\hline CM-058 & $68 / F$ & Michoacan, Mexico & No & - & + & - & NP \\
\hline CM-082 & $70 / F$ & Tegucigalpa, Honduras & Yes & + & + & + & + \\
\hline CM-116 & $34 / \mathrm{M}$ & Acapulco, Mexico & No & Faint positive & - & - & NP \\
\hline CM-121 & $77 / \mathrm{M}$ & Maracay, Venezuela & Yes & + & + & + & + \\
\hline CM-143 & $42 / \mathrm{M}$ & San Miguel, El Salvador & Yes & + & + & + & + \\
\hline CM-155 & $73 / \mathrm{M}$ & Unreported $\ddagger$ & No & Faint positive & - & - & NP \\
\hline CM-174 & 78/M & Guerrero, Mexico & Yes & + & + & + & + \\
\hline CM-197 & $62 / \mathrm{M}$ & Tamaulipas, Mexico & No & + & - & - & NP \\
\hline CM-243 & $54 / \mathrm{M}$ & Durango, Mexico & No & Faint positive & - & - & NP \\
\hline
\end{tabular}

*All patients were of White race and Latinx ethnicity. A total of 83 patients tested negative by STAT-PAK (Chembio Diagnostic Systems, Inc.,

https://chembio.com) and Hemagen (Hemagen Diagnostics, Inc., https://www.hemagen.com). This table displays the 14 patients who tested positive on

$\geq 1$ of the screener assays, whose samples where then sent to CDC for testing. None of the 83 patients who tested negative by the 2 screening assays

had samples sent to CDC for confirmation testing. BCM, Baylor College of Medicine; CDC, Centers for Disease Control and Prevention; EIA, enzyme

immunoassay; ID, identification; NP, not performed; - , negative; + , positive.

†True positive refers to the CDC guidelines recommending a minimum of $\geq 2$ positive test results using $\geq 2$ different diagnostic assay techniques

(https://www.cdc.gov/parasites/chagas/healthprofessionals/dx.html).

†Participants choose not to answer state, country of birth, or both because of personal concerns. 
their home country. Two were polyparous mothers, and none of their children had been tested for Chagas disease. Only 2 of the 7 patients with Chagas cardiomyopathy had ever heard of Chagas disease, and only 1 of these patients knew how Chagas disease was acquired.

\section{Conclusions}

Our study adds to the growing body of evidence supporting T. cruzi surveillance of Latinx patients with nonischemic cardiomyopathy or other risk factors for T. cruzi infection in the United States. T. cruzi infection accounts for a considerable proportion of nonischemic cardiomyopathy in foreign-born Latinx patients $(7 \%-19 \%)(4,5)$, and the timely diagnosis of their infection is imperative.

Our investigation has a few limitations, including the inability to perform additional cardiac imaging and diagnostic studies or follow patients long-term to evaluate prospective identification of underlying etiology. As highlighted by our discordant results, further work is needed to develop a highly specific diagnostic test to prevent clinical confusion regarding accurate disease status. Determining the underlying etiology has a benefit for Chagas cardiomyopathy patients despite the limited efficacy of treatment with antiparasitics (benznidazole and nifurtimox). Patients with Chagas cardiomyopathy might be recommended for heart transplant (10) and can positively respond to implantable cardioverter-defibrillator placement (11) and amiodarone (12). Awareness of infection could lead to testing of at-risk family members who might respond favorably to early treatment.

\section{Acknowledgments}

We thank Kaila Fagerstrom for assisting with patient recruitment.

This project was funded by the National Institutes of Health, National Institute of Allergy and Infectious Diseases (grant no. R21 AI114877-01).

\section{About the Author}

Dr. Nolan is an assistant professor of epidemiology at the University of South Carolina's Arnold School of Public Health. Her research program focuses on the clinical epidemiology of vectorborne and parasitic diseases of the Americas.

\section{References}

1. Bern C, Messenger LA, Whitman JD, Maguire JH. Chagas disease in the United States: a public health approach. Clin Microbiol Rev. 2019 Nov 27;33(1):e00023-19. doi: 10.1128/ CMR.00023-19. PMID: 31776135

2. Pino-Marín A, Medina-Rincón GJ, Gallo-Bernal S, Duran-Crane A, Arango Duque ÁI, Rodríguez MJ, Medina-Mur R, Manrique FT, Forero JF, Medina HM. Chagas cardiomyopathy: from Romaña sign to heart failure and sudden cardiac death. Pathogens. 2021 Apr 22;10(5):505. doi: 10.3390/pathogens10050505. PMID: 33922366

3. Manne-Goehler J, Umeh CA, Montgomery SP, Wirtz VJ. Estimating the burden of Chagas disease in the United States. PLoS Negl Trop Dis. 2016;10:e0005033. https://doi.org/10.1371/journal.pntd.0005033

4. Manne-Goehler J, Reich MR, Wirtz VJ. Access to care for Chagas disease in the United States: a health systems analysis. Am J Trop Med Hyg. 2015;93:108-13. https:/ / doi.org/10.4269/ajtmh.14-0826

5. Stimpert KK, Montgomery SP. Physician awareness of Chagas disease, USA. Emerg Infect Dis. 2010;16:871-2. https://doi.org/10.3201/eid1605.091440

6. Traina MI, Sanchez DR, Hernandez S, Bradfield JS, Labedi MR, Ngab TA, et al. Prevalence and impact of Chagas disease among Latin American immigrants with nonischemic cardiomyopathy in Los Angeles, California. Circ Heart Fail. 2015;8:938-43. https:/ / doi.org/10.1161/ CIRCHEARTFAILURE.115.002229

7. Kapelusznik L, Varela D, Montgomery SP, Shah AN, Steurer FJ, Rubinstein D, et al. Chagas disease in Latin American immigrants with dilated cardiomyopathy in New York City. Clin Infect Dis. 2013;57:e7. https:/ / doi.org/ 10.1093/cid/cit199

8. Bustamante DM, De Urioste-Stone SM, Juárez JG, Pennington PM. Ecological, social and biological risk factors for continued Trypanosoma cruzi transmission by Triatoma dimidiata in Guatemala. PLoS One. 2014;9:e104599. https://doi.org/10.1371/journal.pone.0104599

9. Rabinovich JE, Gürtler RE, Leal JA, Feliciangeli D. Density estimates of the domestic vector of Chagas disease, Rhodnius prolixus Stål (Hemiptera: Reduviidae), in rural houses in Venezuela. Bull World Health Organ. 1995;73:347-57.

10. Benatti RD, Oliveira GH, Bacal F. Heart transplantation for Chagas cardiomyopathy. J Heart Lung Transplant. 2017;36:597-603. https:/ / doi.org/10.1016/j.healun.2017.02.006

11. Pavão MLRC, Arfelli E, Scorzoni-Filho A, Rassi A Jr, PazinFilho A, Pavão RB, et al. Long-term follow-up of Chagas heart disease patients receiving an implantable cardioverterdefibrillator for secondary prevention. Pacing Clin Electrophysiol. 2018;41:583-8. https:/ / doi.org/10.1111/pace.13333

12. Stein C, Migliavaca CB, Colpani V, da Rosa PR, Sganzerla D, Giordani NE, et al. Amiodarone for arrhythmia in patients with Chagas disease: a systematic review and individual patient data meta-analysis. PLoS Negl Trop Dis. 2018;12:e0006742. https://doi.org/10.1371/journal.pntd.0006742

Address for correspondence: Melissa Nolan, University of South Carolina, 915 Greene St, Ste 435D, Columbia, SC 29208, USA; email: msnolan@mailbox.sc.edu 